\title{
Psychological Outcomes in Disaster Responders: A Systematic Review and Meta-Analysis on the Effect of Social Support
}

\author{
Johnrev Guilaran ${ }^{1,2} \cdot$ Ian de Terte $^{1} \cdot$ Krzysztof Kaniasty $^{3,4} \cdot$ Christine Stephens $^{5}$
}

Published online: 20 September 2018

(C) The Author(s) 2018

\begin{abstract}
Disaster response work is associated with various psychological outcomes. In post-disaster conditions, social support is generally observed to impact mental health, particularly for survivors. This review was conducted to survey the extent of social support effectiveness on disaster responder groups. Published quantitative social support studies on police, emergency medical responders, rescue and recovery workers, firefighters, and military responders were searched in various academic databases using keyword searches, a reference list search, and a citation search that resulted in 24 studies with 90 effect sizes being included in the final data base. Articles were coded and effect sizes were averaged using the HedgesVevea Random Effects model. Nineteen categories of psychological outcomes (for example, anxiety, depression, posttraumatic stress symptoms, and psychological distress) and eight classifications of support were coded. Social support was found to be associated with anxiety, burnout, depression, job control, job satisfaction, psychological distress, turnover intentions, and work engagement, with mean effect sizes from -0.36 to 0.57 . Most studies measured perceived social support and negative outcomes.
\end{abstract}

Johnrev Guilaran

John.Guilaran.1@uni.massey.ac.nz

1 School of Psychology, Massey University, Wellington 6021, New Zealand

2 Division of Social Sciences, University of the Philippines Visayas, Miagao, Iloilo 5023, Philippines

3 Department of Psychology, Indiana University of Pennsylvania, Indiana, PA 15705, USA

4 Polish Academy of Sciences, 00-378 Warsaw, Poland

5 School of Psychology, Massey University, Palmerston North 4474, New Zealand
Social support correlated with outcomes in police responders and rescue and recovery workers. This review discusses the breadth of effect of social support, as well as other elements, such as temporal factors, that may affect the effectiveness of social support in disaster responders.

Keywords Disaster responders · Meta-analysis · Postdisaster psychological outcomes · Psychological distress - Social support

\section{Introduction}

Disasters are collective experiences that affect people at the community and individual levels. Exposure to these events is associated with both negative (Bonanno et al. 2010; Goldmann and Galea 2014; Fullerton et al. 2015) and positive (Bonanno et al. 2010; Harada et al. 2015) psychological outcomes, which are observed in the general affected population. These outcomes are also observed in individuals who respond and provide assistance in the aftermath of disaster events (Benedek et al. 2007; Fullerton et al. 2013; Bromet et al. 2016). In studies that have investigated the correlates of these psychological outcomes, social support is found to be one of the most reliable factors associated with fewer negative and more positive outcomes. Hobfoll and Stokes (1988)—and later, Kaniasty and Norris (2009)-highlighted three facets of this construct: (1) receipt of actual assistance; (2) perception of availability of support; and (3) integration in a network of caring individuals. These facets of support are viewed to make unique contributions to psychological outcomes in the aftermath of disastrous events.

Notably, the Social Support Deterioration Deterrence (SSDD) model developed by Kaniasty and Norris 
(1993, 1995, 2009) suggests that perceived social support directly affects psychological outcomes while received social support influences perceptions of support; thus, receipt of support indirectly affects psychological outcomes. The SSDD model also posits that mobilization and utilization of social support are unequal and inequitable in times of disasters. Mobilization of social support is influenced by predisaster factors such as social status and other resources, which dictate the relative advantage/disadvantage in receiving social support. Although people with more severe exposure to disasters are typically expected to experience more psychological distress, they are also likely to receive more social support because they are perceived to need it more than those with less severe disaster exposure.

That social support is beneficial in the aftermath of disasters is well documented (Norris et al. 2002; Norris and Elrod 2006), but the degree to which it is beneficial for disaster responders is yet uncharted. Disaster responders are professionals tasked with the "protection and preservation of life, property, and the environment" (Prati and Pietrantoni 2010, p. 403) in the aftermath of catastrophic events. Aside from being support providers, these individuals are also support recipients. In addition, responders usually operate under a structure that embeds them in a group of individuals with shared experiences. Thus, in terms of social support, disaster responders have the unique context of systematically providing support while arguably systematically receiving support themselves. The gap lies in knowing how these support-related circumstances affect the association between social support and psychological outcomes.

This article presents a general picture of social support investigations among disaster responders. Social support is considered as one of the cornerstones of psychological recovery (Hobfoll et al. 2007), where increase in support is usually associated with lower risk for psychopathology (Goldmann and Galea 2014). But the effectiveness of social support is influenced by several factors, such as the sources of support (Halbesleben 2006) and culture (Chen et al. 2012). Temporal elements are also crucial in the context of disasters: social support is observed to deteriorate over time (Kaniasty and Norris 1995). This article identifies the different psychological outcomes associated with social support in disaster responder groups, and summarizes the strength of social support-outcome associations, while also considering some of the influencing factors mentioned earlier in this paragraph.

A number of meta-analyses have shown the link between social support and psychological outcomes. Metaanalyses on the correlates of posttraumatic stress disorder (PTSD) showed lack of social support as a risk factor, and having social support as a protective factor (Brewin et al. 2000; Ozer et al. 2003). These studies only focused on PTSD, however, and did not specifically target social support in disaster first responders. The meta-analysis by Prati and Pietrantoni (2010), on the other hand, targeted social support and first responder mental health outcomes. The current study differs from previous work in three major aspects: (1) our work specifically explores the strength of association of social support and psychological outcomes in the context of disasters; (2) this study casts a wider net in terms of how social support is defined and how psychological outcomes are covered; and (3) we attempt to synthesize these associations in terms of facets of support, type of responder, type of outcome, and support and outcome measurement time lags.

\section{Article Selection and Inclusion}

Articles were identified through several methods (Fig. 1). A Boolean keyword search in PsycINFO $(n=138)$, PubMed $(n=276)$, and Web of Science $(n=193)$ was performed using the social support keywords: "social support," "received support," "perceived support," "social embeddedness," "social integration," "emotional support," “informational support," "practical support," "instrumental support," "social network," and "assistance"; responder keywords: "emergency first responder," "first responder," “emergency responder," "emergency worker," “police," “military," “fire fighter," "ambulance," "red cross," "red crescent," and "disaster responder"; and "disaster*." The 607 abstracts were then screened using the following criteria: (1) they must be quantitative studies on disaster first responders; (2) each must explicitly measure social support; (3) every study must measure at least one psychological outcome; and (4) the studies selected must also be carried out in the context of a "disaster", which was defined using the Emergency Database (EM-DAT) definition (Guha-Sapir et al. 2016). Studies that did not meet these criteria were excluded. This reduced the data base to 20 articles. After removing eight duplicates, the remaining articles were added to the 10 articles at hand to constitute an initial article pool. The reference lists of these 22 articles were examined to search for additional studies for possible inclusion. No new studies were found using this method. Using Google Scholar, manuscripts that cited the screened studies were checked for possible inclusion in the review. This resulted in 13 additional articles. One manuscript was excluded due to non-response from the author upon request for the full text. The full-text articles were then inspected for eligibility using the same criteria used in the screening procedure, and 10 potential articles were excluded, which resulted in 24 studies being included in the final review.

Effect sizes on formal support, such as debriefing and psychotherapy, were excluded because this article is 
Fig. 1 Process of article search, screening, and inclusion (Moher et al. 2009) of studies used in a meta-analysis of psychological outcomes in disaster responders. Screening of the manuscripts used the following inclusion criteria: (1) quantitative studies on disaster responders; (2) studies that explicitly measure social support; (3) studies that measure at least one psychological outcome; and (4) studies in the context of a disaster. The following exclusion criteria were applied: (1) studies where social support was in the form of formal support, such as psychotherapy; and (2) studies where social support is the outcome rather than the predictor

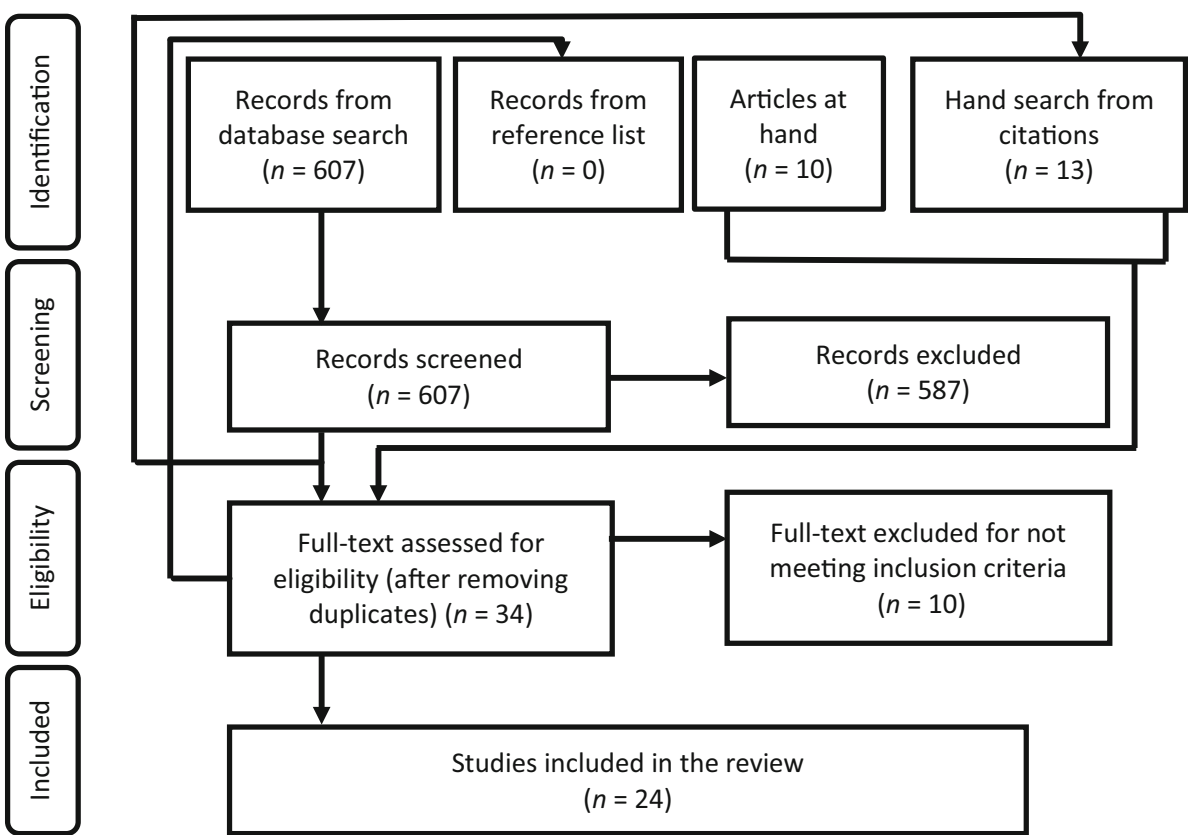

focused on social support from nonprofessional support providers. Also excluded are studies where social support is the outcome variable. An exception was made for Schwarzer et al. (2016), where social support and the psychological outcome variables were measured at the same time, despite social support being framed as an outcome.

\section{Coding of Articles}

Articles were coded according to year of publication, responder/sample, sample size, disaster, social support measure, outcome measure, and effect size. Responder types were clustered into five based on the number of studies: emergency medical responders; firefighters; police; rescue and recovery workers; and, others, which includes military responders and disaster responders that were aggregated (for example, combined police, firefighters, and emergency medical responders). Social support measures were then categorized according to the facet of support: general/undifferentiated social support, received social support, perceived social support, social support need, social support utilization, lack of support, and negative support. Measures of frequency of contact, time spent with others, and those that are relationship-based were coded as general/undifferentiated support. Social support-seeking and social-support coping were coded under support utilization. Outcomes were also coded as positive or negative psychological outcomes. Absence or reduction of negative outcomes/symptoms were coded as positive outcomes.

Variations in the time lag between the disaster occurrence and the measurements were observed. This prompted the addition of two codes. First is the disaster-social support measurement time lag, which is the number of months between the disaster and the measurement of social support. A pre-disaster measurement was given a negative code (for example, measurement at 10 months before the disaster was coded "-10"). Another is the social supportoutcome measurement time lag, which is the number of months between the measurement of social support and the psychological outcomes. Studies where social support and the psychological outcome were measured concurrently were given a code of " 0. " In cases where measurement spanned for several months, or was done in two time points, the median number of months was derived. One week was coded as " $0.25 " ; 3$ weeks, "0.75."

\section{Calculation of Effect Sizes and Method of Meta- analysis}

A significant number of studies included in the review have multiple measures of social support and psychological outcomes, some with measurements in more than one time points. A unique combination of social support type, psychological outcome, and social support/outcome measurement time lag was considered one effect size. Within study effect sizes were combined using the Fixed-Effects model (Hedges and Olkin 1985; Hedges and Vevea 1998) because this method limits the generalizability of the combined effect only to the study sample. The meta-analyses of the different study effect sizes were conducted using the Hedges-Vevea Random Effects model (Hedges and Vevea 1998) as, in contrast to the Fixed-Effects model, combined effect sizes 
using this approach allows for generalization of effects to populations outside the study. The Pearson Product Moment Correlation Coefficient was used as the base effect size as it was the most common effect size across the different studies, and is less prone to interpretation error (Field and Gillett 2010). Odds ratios were transformed to Pearson's r correlation coefficient using Eq. 1 (Field and Gillett 2010), where $r=$ Pearson correlation coefficient and $O R=$ odds ratio. Beta weights were converted to Pearson's $r$ correlation coefficient using Eq. 2, which was derived from Eq. 3 (Gardner 2010), where $t=\frac{b}{S E_{b}}, r^{2}$ is the overall coefficient of determination, $N$ is the sample size, and $p$ is the number of predictors,

$r=\frac{O R^{0.5}-1}{O R^{0.5}+1}$

$r=\sqrt{\frac{\left(\frac{b}{S E_{b}}\right)^{2}\left(1-R^{2}\right)}{N-p-1}}$

$r^{2}=\frac{t^{2}\left(1-R^{2}\right)}{N-p-1}$

Heterogeneity of effect sizes was tested using the Chi square test of homogeneity, with $d f=n-1$. Data were analyzed using Field and Gillett's (2010) SPSS syntax.

\section{Results}

Twenty four studies with 90 effect sizes were included in the final analysis (for a summary, see Table 1). Publication years ranged from 1995 to 2017, with more than 50\% of the studies published after 2010. Police officers were the most researched disaster responders, studied by more than $76 \%$ of the studies reviewed. Thirty-three percent of the studies were on the 9/11 Attack, making it the most studied disaster. More than half of the studies were conducted in the United States.

Measurement of post-disaster social support ranged from 1 week after the event to more than 10 years after. Only two studies had pre-disaster social support measures. Most of the studies had concurrent measures of social support and psychological outcome, with only six studies having a time lag ranging from 3 weeks to more than 10 years. Considering the wide variation in measurement time lags, separate meta-regression analyses were performed on disaster-social support measurement time lag and social support-outcome measurement time lag. No relationship was found between measurement time lag and effect size.

Almost half of the studies measured perceived social support, making it the most studied facet of social support in this review. This was followed by general/undifferentiated support with five studies and 10 effect sizes, and support utilization with four studies and 10 effect sizes. Nineteen categories of psychological outcomes were observed. Of the 90 obtained effect sizes, only 14 were associations between social support and positive outcomes, such as resilience and posttraumatic growth. The other 76 effect sizes included normative negative outcomes, such as turnover intentions and perceptions of job demands; general psychological distress that may indicate psychopathology; or clinical outcomes such as posttraumatic stress symptoms (PTSS), depression, and anxiety. PTSS and psychological distress are the most studied outcomes. A summary of the number of effect sizes per social support facet and psychological outcome is found in Table 2.

To obtain the overall magnitude of social support effectiveness on psychological outcomes, a meta-analysis on the absolute value of the 90 effect sizes was performed. This resulted in a weighted average effect size of $0.19(p<0.001)$ with a Fail-Safe $\mathrm{N}$ of 50,293. Effect sizes were found to be homogeneous. But it is not assumed that positive and negative psychological outcomes fall on the same continuum; hence, separate meta-analyses were conducted on each of the outcome categories (Table 3). Compassion satisfaction, gratitude, job control, job satisfaction, life satisfaction, posttraumatic growth, resilience, work engagement, and reduction of posttraumatic stress symptoms were coded as positive outcomes, and all others coded as negative outcomes. Effect sizes were synthesized according to the five clusters of responders specified in the previous section. Social support was found to have an effect on both positive $(\bar{r}=0.39, p<0.001)$ and negative psychological outcomes $(\bar{r}=-0.15, p<0.005)$ in the police, and on negative outcomes $(\bar{r}=-0.27, p<0.001)$ in search and rescue workers and other responders $(\bar{r}=-0.19, p<0.001)$, with small to medium effect sizes. The 13 effect sizes associated with positive outcomes in police responders came from only three studies. Effect sizes associated with negative outcomes in rescue workers and other responders came from four studies each.

Syntheses of effect sizes according to type of social support show perceived support to be associated with positive psychological outcomes $(\bar{r}=0.41, p<0.001)$ and negative outcomes $(\bar{r}=-0.2, p<0.001)$, and received support with negative outcomes $(\bar{r}=-0.24, p<0.001)$. Fail-Safe $\mathrm{N}$ for received support indicates that the estimates are not robust. The 13 effect sizes involved in estimating the average effect size of perceived support on positive outcomes were obtained from just three studies.

Effect sizes on social support associations with specific psychological outcomes were pooled according to measurement time lag between the two variables (Table 4). This analysis focused on the effect sizes of presence of 


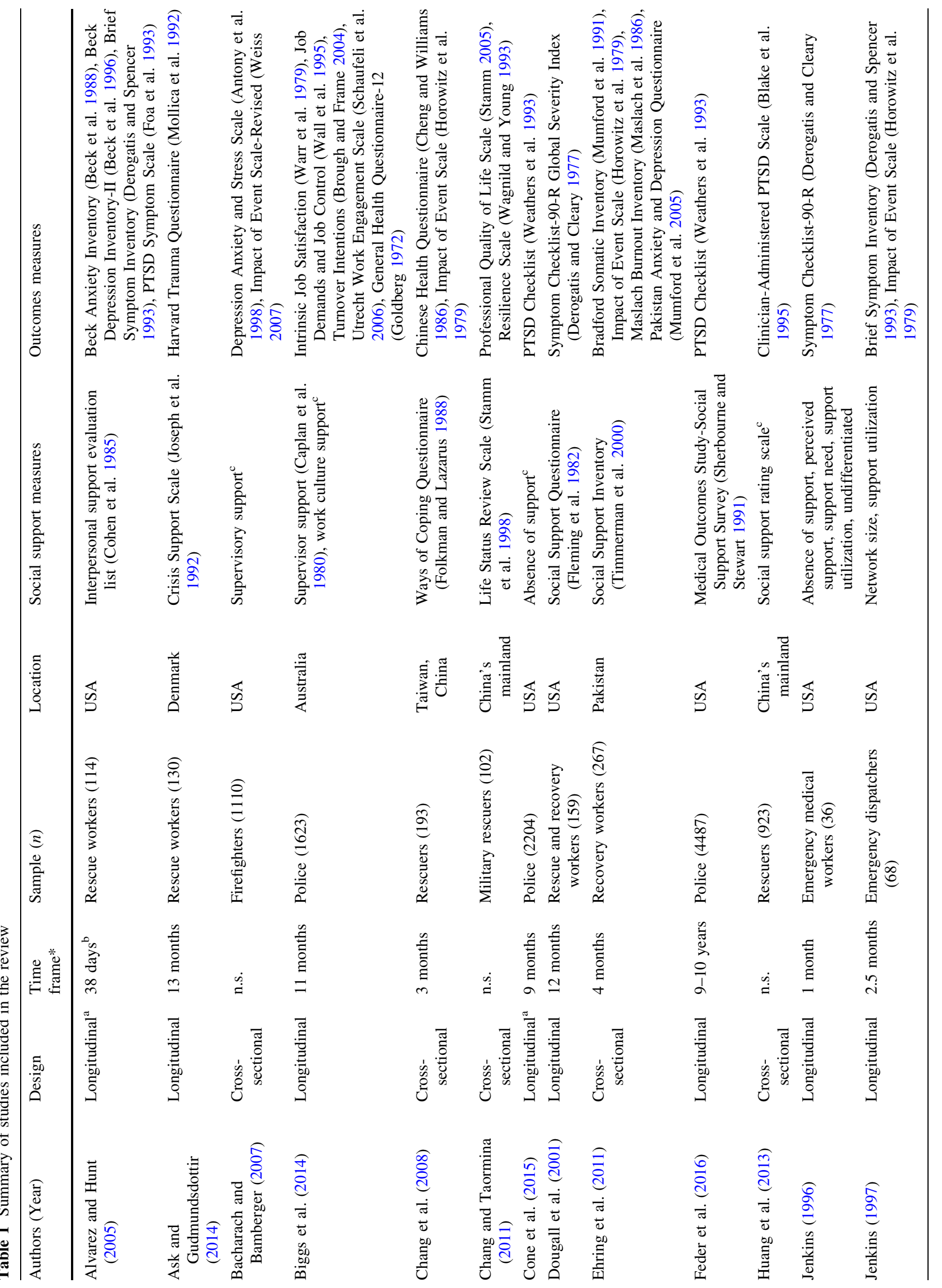




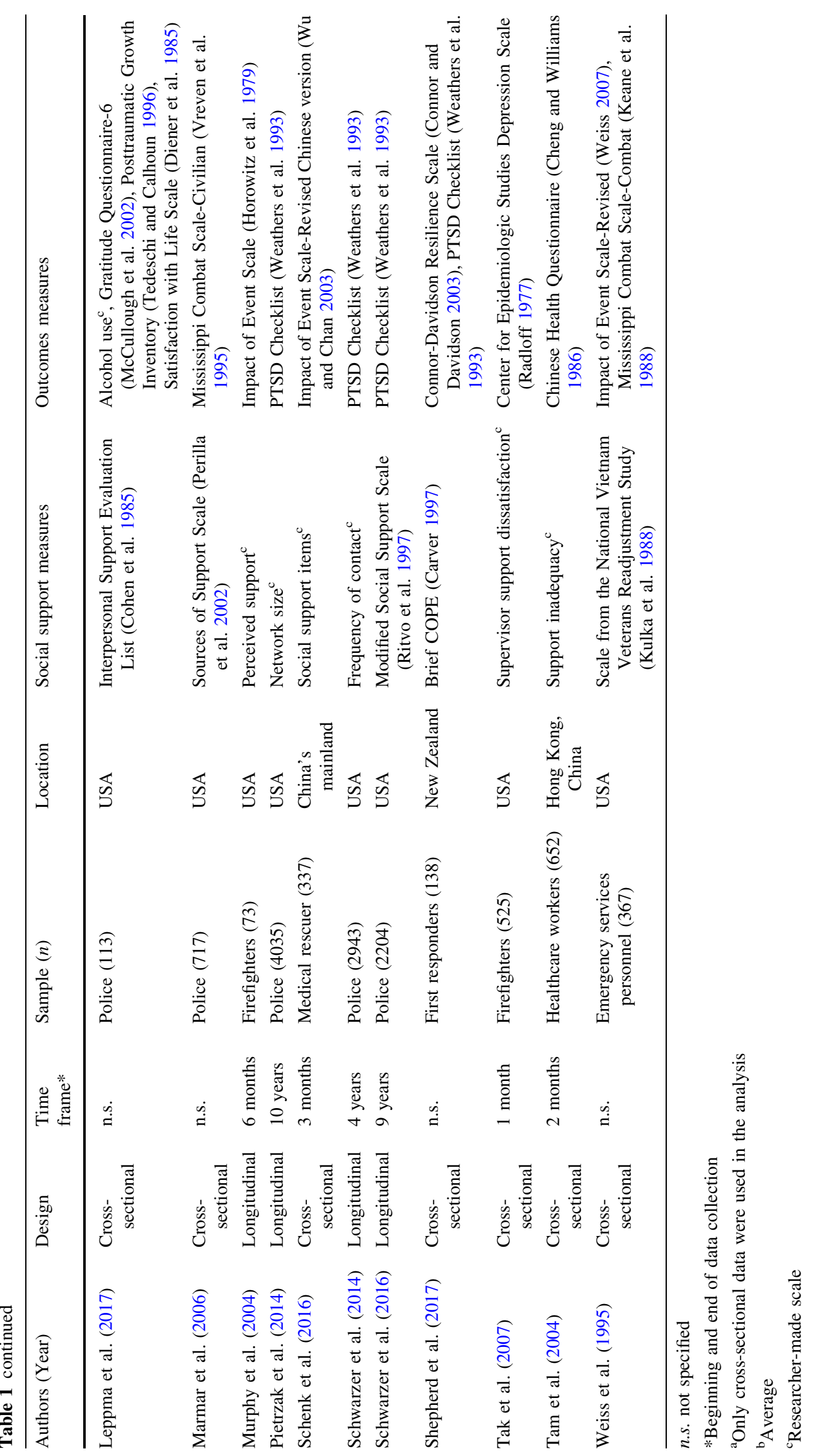


Table 2 Number of effect sizes of associations of social support type and psychological outcomes in disaster responders

\begin{tabular}{|c|c|c|c|c|c|c|c|c|c|}
\hline Psychological outcomes & GenSS & RSS & PSS & Net & Use & Need & Abs & NegSS & Total no. of ES \\
\hline Alcohol use & & & 1 & & & & & & 1 \\
\hline Anxiety & 1 & & 4 & & 1 & 1 & 1 & & 8 \\
\hline Burnout & 1 & & 1 & & & & & & 2 \\
\hline $\mathrm{CS}$ & 1 & & & & & & & & 1 \\
\hline Depression & 1 & & 4 & & 1 & 1 & 1 & 1 & 9 \\
\hline Gratitude & & & 1 & & & & & & 1 \\
\hline Hostility & 1 & & 1 & & 1 & 1 & 1 & & 5 \\
\hline Job control & & & 3 & & & & & & 3 \\
\hline Job demands & & & 3 & & & & & & 3 \\
\hline Job satisfaction & & & 3 & & & & & & 3 \\
\hline Life satisfaction & & & 1 & & & & & & 1 \\
\hline OC symptoms & 1 & & 1 & & 1 & 1 & 1 & & 5 \\
\hline Psych. distress & 1 & 1 & 10 & 1 & 2 & & & 1 & 16 \\
\hline PTG & & & 1 & & & & & & 1 \\
\hline PTSS & 2 & 1 & 9 & 3 & 3 & & 4 & & 22 \\
\hline Resilience & 1 & & & & 1 & & & & 2 \\
\hline Stress & & & 1 & & & & & & 1 \\
\hline Turnover intentions & & & 3 & & & & & & 3 \\
\hline Work engagement & & & 3 & & & & & & 3 \\
\hline Total no. of ES & 10 & 2 & 50 & 4 & 10 & 4 & 8 & 2 & 90 \\
\hline
\end{tabular}

CS compassion satisfaction, GenSS general/undifferentiated social support, $R S S$ received social support, $P S S$ perceived social support, Net social integration/embeddedness and network size, Use support utilization and coping, Need social support need, Abs absence of support, NegSS negative social support, $O C$ obsessive-compulsive, $P T G$ posttraumatic growth, $P T S S$ posttraumatic stress symptoms, ES effect size

support on the outcomes. Negative support, support need, absence of support, and support utilization were excluded as these facets of support belong to a different taxonomy; in addition, previous analyses have shown that these factors have no effect on psychological outcomes. All 19 outcomes had concurrent measures, while only 10 outcomes had a time difference between social support measurement and outcome measurement, allowing for an observation of effects of social support across time.

Consistent with the previous analyses, concurrent associations show social support to have the largest effect sizes on positive outcomes: job satisfaction $(\bar{r}=0.57, p<0.001)$ and work engagement $(\bar{r}=0.42, p<0.001)$. Work-related outcomes also have larger effect sizes than the other psychological outcomes. Anxiety $(\bar{r}=-0.19, p<0.001)$ and psychological distress $(\bar{r}=-0.32, p<0.001)$ are the only clinical outcomes associated with social support. Furthermore, psychological distress is the only outcome for which a time-lagged effect of social support was observed $(\bar{r}=-0.2, p<0.001)$.

\section{Discussion}

Responding to disasters takes a psychological toll on the responder, and common knowledge suggests the benefits of social support in these circumstances. This review shows that although having social support is helpful, the benefits of social support are within bounds. This is, first and foremost, shown by effect sizes that are small to medium, leaving a large amount of variance in psychological outcomes that cannot be explained by social support. In addition, the effects of social support on psychological outcomes were observed in some conditions but not in others, which suggests that the psychological benefits of social support are not absolute.

Studies included in the review utilized a wide variety of instruments to measure social support. One explanation is that researchers may have a different understanding of what constitutes social support. Distinctions between the different facets of support are imperative, as each facet has a unique contribution to psychological outcomes. These effects are also magnified by disasters. These types of critical events challenge resources, including social resources such as social support. The Social Support Deterioration Deterrence (SSDD) model suggests that in the aftermath of disasters, people have unequal and 


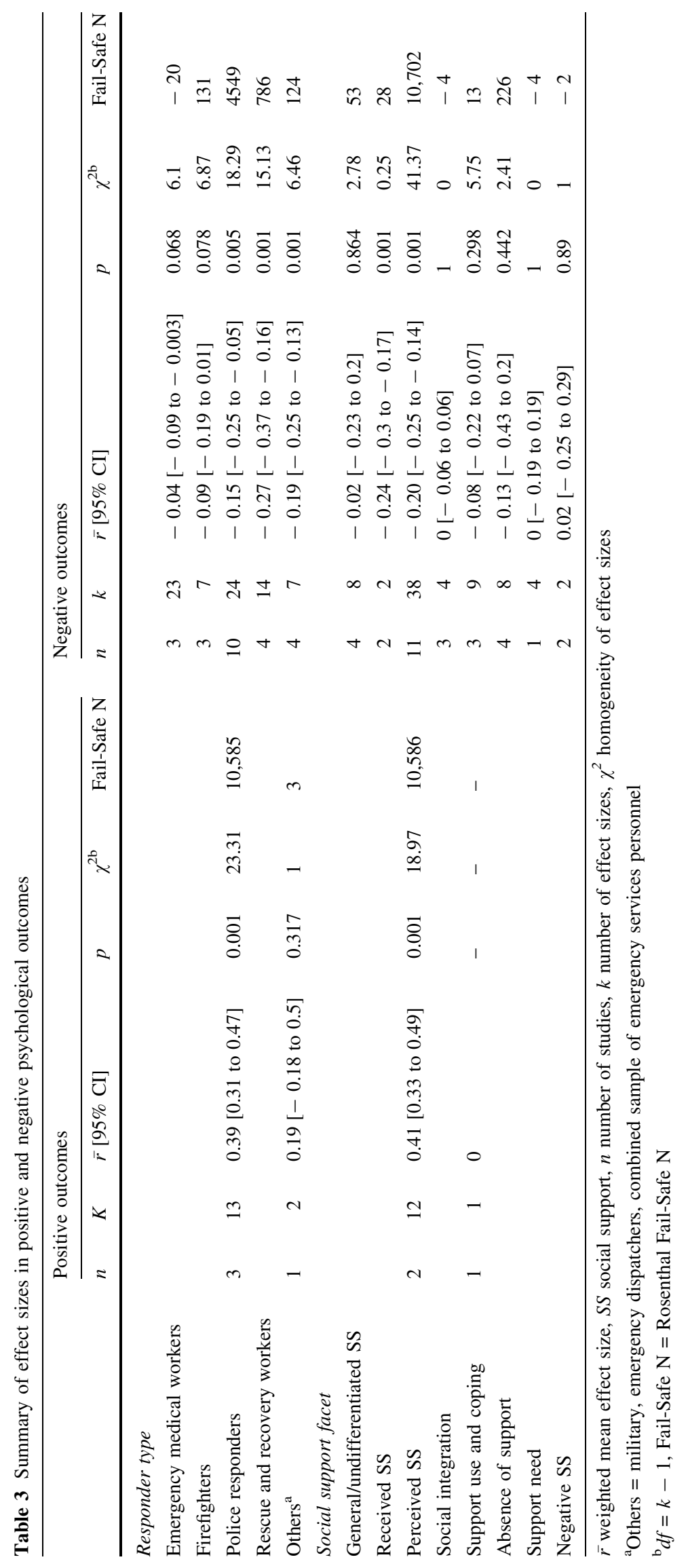




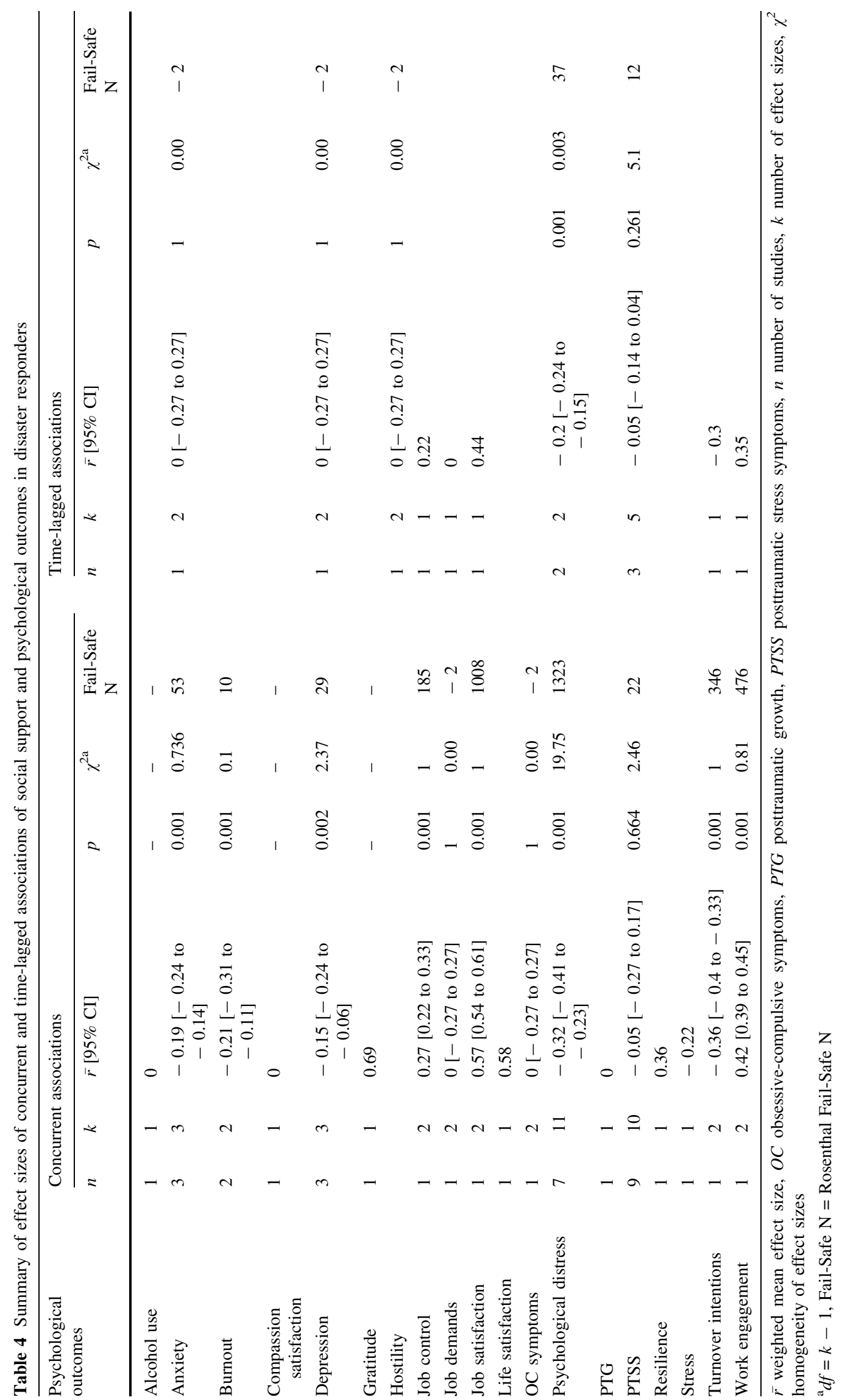


Fig. 2 Absolute value of reported effect sizes $(k=147)$ across social supportpsychological outcome measurement time lag (months)

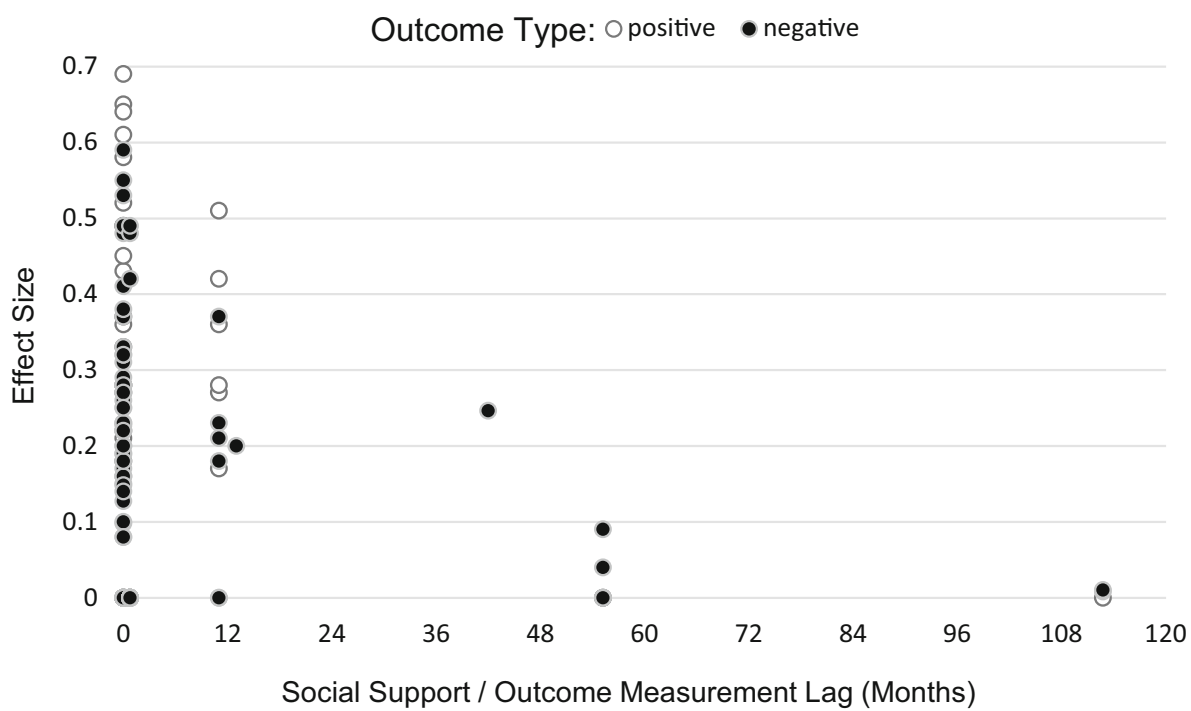

inequitable access to and utilization of support, which may, in turn, influence people's perception of support (Kaniasty and Norris 2009). Perceptions of support directly influence emotional distress but receipt of actual support may only have indirect effects. People who receive support may also not necessarily feel supported, as explained by the StressSupport Matching Hypothesis (Curtona and Russell 1990), which suggests that support is only effective if it answers the need. Furthermore, the Social Support Effectiveness model (Rini and Dunkel Schetter 2010) suggests that whether social support is helpful or harmful depends on the degree to which the particular supportive behaviors address the need in terms of both quality and quantity. These models of explaining social support dynamics, which are anchored on empirical observations, highlight the need to study social support not as a global construct, but as a multidimensional one.

Having stated this, it is worth noting that the majority of the studies focused on perceived social support. Congruent with the SSDD model, perceived support-having direct effects on psychological outcomes-has the largest effect size among the facets of support. Perceived support comes in the form of appraisal of support quality and availability, and it has long been shown that appraisal of risk and protective factors such as social support in the context of disasters is closely associated with post-disaster outcomes (Bonanno et al. 2010). Such forms of appraisal are also found to be clinically useful in treating post-disaster psychological distress, as in the case of cognitive behavioral therapies (Hamblen et al. 2017). However, it is also important to study more concrete facets of support-received support and social embeddedness, which can be externally controlled as a form of intervention.
Other than support facet, the effect size of social support on psychological outcomes also varied across type of responder. The observed effect size in police responders has been corroborated by social support studies on police officers outside the disaster context as well (Stephens et al. 1997; de Terte et al. 2014). The absence of observed effect in other clusters of responders, however, does not necessarily mean social support is ineffective in these groups. These results must be interpreted in the context of small numbers of studies, differences in support measures, and other moderating variables that are not accounted for in this review.

In spite of the small number of effect sizes involved, it is important to note that social support affects work-related psychological outcomes at medium to large effect sizes. Work-related outcomes are normative, as opposed to clinical outcomes. They are also less intense than clinical outcomes, which could require professional help, such as psychotherapy. What these results suggest is that the effectiveness of social support decreases as the psychological outcome becomes more clinical in nature. It is clear that social support has the potential to alleviate symptoms, but given the present evidence, it should not replace the more specialized forms of treatment of clinical syndromes in disaster responders. This demonstrates one of the limitations of social support effectiveness.

Differences in the effect sizes of social support in psychological outcomes were also observed between concurrent and time-lagged measurements, where effect sizes in time-lagged measurements were lower than those in concurrent measurements, which runs contrary to the findings of Prati and Pietrantoni (2010). This was observed both at the individual study level (for studies with time-lagged measurements) and at the meta-analytic level. To test this 
Fig. 3 Absolute value of reported effect sizes $(k=88)$ of concurrent measurements across disaster-social support measurement time lag (months)

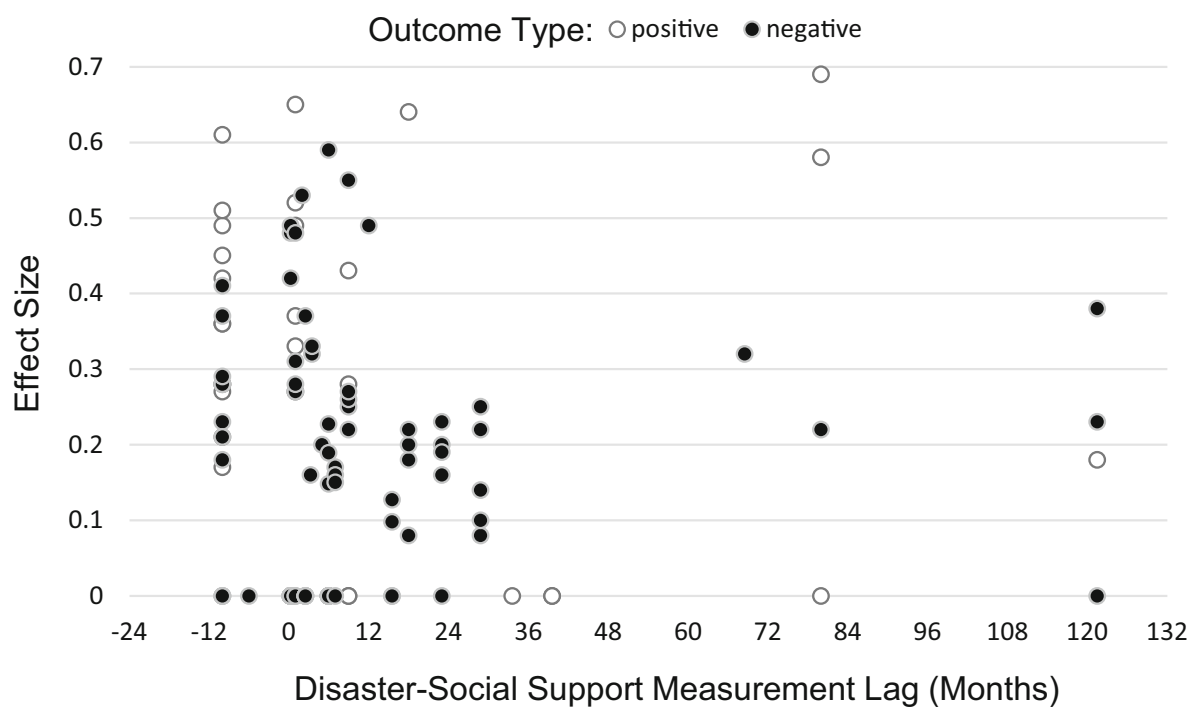

observation further, the absolute values of the 147 reported effect sizes (before they were combined at the individual study level) were plotted against the lag between the measurement of social support and of the psychological outcomes (Fig. 2), where a pattern of effect sizes approaching zero is observed as the lag increases. Admittedly, there are very few studies included with longer support / outcome measurement time lags, which possibly accounts for the absence of correlation between the two variables.

In the same fashion, the absolute values of the 88 reported effect sizes on concurrent measurements were plotted against the time of measurement relative to the disaster (Fig. 3) in order to check for patterns of effect size changes in concurrent measurements. This generated a more dispersed scatterplot, but further inspection revealed a pattern of effect size reduction from 10 months prior to disaster until 28.8 months after, with a correlation approaching significance $(k=71, r=-0.23, p=0.054)$. Furthermore, effect sizes 80 months post-disaster seem to follow the pre-disaster effect size dispersion pattern. With a small number of effect sizes involved and with the innate limitations of meta-analytic reviews, these observations are far from conclusive. But they support the idea of postdisaster social support deterioration. In addition, support deterioration may possibly naturally discontinue sometime around 28.8 months after the disaster. These observations are worth looking into in future studies, as it is crucial to know patterns of decline and rebound of social support effectiveness in order to know when to intervene.

Individual studies have long observed the long-term effects of social support on psychological outcomes (Holahan and Moos 1981; Kaniasty and Norris 2008), but the deterioration of effect over time has not yet been thoroughly studied. This brings to light another possible property of social support: its effectiveness and relevance may decrease over time. This is especially important in the context of disasters, where social support is observed to deteriorate over time as revealed by the SSDD model employed by Kaniasty and Norris (2009). Along with the deterioration of support is the possible deterioration of its effect. This is not very surprising but should be pointed out, nevertheless. Social support has long-term effects on psychological outcomes, and the strength of these effects may depend on when the support is provided.

The results further the debate on the role that social support plays in effecting psychological change. Traditionally, social support is framed to have main effects or stressbuffering effects on psychological outcomes (Cohen et al. 2000). The main effects model suggests that even in the absence of stressful events, social support contributes to positive outcomes. The stress-buffering model, on the other hand, suggests that social support in itself has little effect on mental health in times of calm whereas it substantially reduces the negative impact of stressful experiences such as disasters. This effect was observed in this review: presence of support was linked to reduction of negative outcomes, and absence of support had a non-significant mean effect size.

On the other hand, social support being positively associated with favorable psychological outcomes after disaster exposure does not fit the stress-buffering frame. However, it fits a positive outcome-enhancement frame. Both buffering and enhancement effects are statistical moderation patterns (Jose 2013), and with the assumption that the effect sizes observed are conditional to the disaster exposure, social support may enhance positive outcomes and buffer the negative effects of disaster exposure that results in lower levels of negative outcomes. In the absence of pre-disaster measures, this is speculation, but is worth exploring further. 
It is interesting to note that our results differ from those of Brewin et al. (2000) and Ozer et al. (2003) in terms of the association of social support with PTSD. These authors reported weighted effect sizes of 0.43 and -0.28 , respectively compared to finding no effect in this metaanalysis. However, Brewin et al.'s work synthesized effect sizes of lack of support in the context of general traumatic experiences. On the other hand, the current study focuses its analysis on the presence of support on a group of professionals impacted by a specific form of traumatic exposure. Similar to the current analysis, Ozer et al.'s work analyzed the effect sizes of the presence of support, but it differs from the current study in two ways. First, it is focused on perceived social support, whereas the current study examined perceived support along with received support, undifferentiated support, and social embeddedness. Second, Ozer et al.'s meta-analysis synthesized the effect sizes of two types of samples: the general population and combat-exposed adults. Just as in Brewin et al.'s metaanalysis, these samples were also exposed to broad types of trauma. These key differences in the inclusion of facet of support, type of sample, and type of exposure may explain why the previous meta-analyses found associations between social support and PTSD, in contrast to the absence of such association found in the present analysis.

This review comes with several limitations. First, there are overlaps in some effect sizes in terms of the sample and measures involved. Second, there is also a wide variation of the number of studies and consequently, effect sizes, involved in the analyses. In addition, some syntheses involved effect sizes as few as two, which impact the accuracy of the estimates. Finally, the studies involved in this analysis heavily focused on the 9/11 attacks; hence, generalization of results to disaster responders in general should be done with caution. The inclusion of the number of studies, the number of effect sizes, and the Rosenthal Fail-Safe $\mathrm{N}$ should indicate the robustness of the analyses and would contextualize the estimates. Because of the nature of the analyses, qualitative studies and a number of quantitative studies were excluded.

Notwithstanding these limitations, this review shows the topography of the research area, which may help inform the territories that need to be charted. In contrast to Prati and Pietrantoni's (2010) work, the present review focuses on responders in the context of disasters. This is an important distinction to make. Disasters are critical events that challenge the coping capacity of communities, which consequently increase reliance on external sources of support. This effect of overwhelming collective internal resources is a distinct characteristic of a disaster, distinguishing it from other forms of critical incidents. This review, therefore, sets itself apart from previous work, such that it examines the strength of association between social support - a form of external resource-and psychological outcomes in disaster responders-people who both provide and receive support - in situations where (social) support is highly needed.

\section{Conclusion}

This study examined the effects of social support on various psychological outcomes in disaster first responders. Social support was observed to have varying degrees of association with these outcomes, which may be contingent on the facet of support and other factors associated with the type of responder and other temporal factors. Along with the evidence for usefulness of support, the limitations of this resource were also presented. With these observed conditions that influence the helpfulness of social support, future studies should look into the facets of support that can be used for intervention, and the conditions that may optimize the effectiveness of these supportive behaviors and interactions.

Social support is spontaneous and naturally occurring. As such, it presents itself as a sustainable form of psychosocial intervention for buffering the negative consequences of disasters in responder groups. As this article illustrates, social support may even enhance positive outcomes in the aftermath of disaster exposure. However, good intentions do not always lead to desirable results, and providing support does not always result in positive psychological consequences. Social support may also benefit some types of disaster responders but not others. These differences may be influenced by several factors, such as differences in organizational structure, organizational culture, and the economic benefits of the profession. Future research should look into how these different variables moderate the effectiveness of supportive interactions.

Studies should also pay careful attention to the different components of social support and explore how these components influence outcomes in different types of responders. For example, researchers should look into the effectiveness of the different forms and sources of social support for police officers. These efforts could then inform the development of social support-based interventions, such as peer support programs or programs that focus on their work partners. It is not only important to know who can support disaster responders, but what form of social support works, and when best to provide these supportive behaviors. Social support is a potent element of post-disaster psychological recovery, but it is important to understand its nuances to optimize its potency. 
Open Access This article is distributed under the terms of the Creative Commons Attribution 4.0 International License (http://crea tivecommons.org/licenses/by/4.0/), which permits unrestricted use, distribution, and reproduction in any medium, provided you give appropriate credit to the original author(s) and the source, provide a link to the Creative Commons license, and indicate if changes were made.

\section{References}

Alvarez, J., and M. Hunt. 2005. Risk and resilience in canine search and rescue handlers after 9/11. Journal of Traumatic Stress 18(5): 497-505.

Antony, M.M., P.J. Bieling, B.J. Cox, M.W. Enns, and R.P. Swinson. 1998. Psychometric properties of the 42-item and 21-item versions of the depression anxiety stress scales in clinical groups and a community sample. Psychological Assessment 10(2): 176-181.

Ask, E., and D. Gudmundsdottir. 2014. A longitudinal study of posttraumatic stress symptoms and their predictors in rescue workers after a firework factory disaster. International Journal of Emergency Mental Health 16(2): 316-321.

Bacharach, S.B., and P.A. Bamberger. 2007. 9/11 and New York City firefighters' post hoc unit support and control climates: A context theory of the consequences of involvement in traumatic workrelated events. Academy of Management Journal 50(4): 849-868.

Beck, A.T., N. Epstein, G. Brown, and R.A. Steer. 1988. An inventory for measuring clinical anxiety: Psychometric properties. Journal of Consulting and Clinical Psychology 56(6): 893-897.

Beck, A.T., R.A. Steer, and G.K. Brown. 1996. Beck depression inventory-II. San Antonio 78(2): 490-498.

Benedek, D.M., C.S. Fullerton, and R.J. Ursano. 2007. First responders: Mental health consequences of natural and humanmade disasters for public health and public safety workers. Annual Review of Public Health 28: 55-68.

Biggs, A., P. Brough, and J.P. Barbour. 2014. Exposure to extraorganizational stressors: Impact on mental health and organizational perceptions for police officers. International Journal of Stress Management 21(3): 255-282.

Blake, D.D., F.W. Weathers, L.M. Nagy, D.G. Kaloupek, F.D. Gusman, D.S. Charney, and T.M. Keane. 1995. The development of a clinician-administered PTSD scale. Journal of Traumatic Stresss 8(1): 75-90.

Bonanno, G.A., C.R. Brewin, K. Kaniasty, and A.M.L. Greca. 2010. Weighing the costs of disaster: Consequences, risks, and resilience in individuals, families, and communities. Psychological Science in the Public Interest 11(1): 1-49.

Brewin, C.R., B. Andrews, and J.D. Valentine. 2000. Meta-analysis of risk factors for posttraumatic stress disorder in trauma-exposed adults. Journal of Consulting and Clinical Psychology 68(5): 748-766.

Bromet, E.J., M.J. Hobbs, S.A.P. Clouston, A. Gonzalez, R. Kotov, and B.J. Luft. 2016. DSM-IV post-traumatic stress disorder among World Trade Center responders 11-13 years after the disaster of 11 September 2001 (9/11). Psychological Medicine 46(4): 771-783.

Brough, P., and R. Frame. 2004. Predicting police job satisfaction and turnover intentions: The role of social support and police organisational variables. New Zealand Journal of Psychology 33(1): 8-18.

Caplan, R.D., S. Cobb, J.R.P. French Jr., R.V. Harrison, and S.R. Pinneau Jr. 1980. Job demands and worker health: Main effects and occupational differences. Ann Arbor, MI: Institute for Social Research.

Carver, C.S. 1997. You want to measure coping but your protocol's too long: Consider the brief cope. International Journal of Behavioral Medicine 4(1): 92-100.

Chang, C.M., L.C. Lee, K.M. Connor, J.R.T. Davidson, and T.J. Lai. 2008. Modification effects of coping on post-traumatic morbidity among earthquake rescuers. Psychiatry Research 158(2): $164-171$.

Chang, K., and R.J. Taormina. 2011. Reduced secondary trauma among Chinese earthquake rescuers: A test of correlates and life indicators. Journal of Loss and Trauma 16(6): 542-562.

Chen, J.M., H.S. Kim, T. Mojaverian, and B. Morling. 2012. Culture and social support provision: Who gives what and why. Personality and Social Psychology Bulletin 38(1): 3-13.

Cheng, T.A., and P. Williams. 1986. The design and development of a screening questionnaire (CHQ) for use in community studies of mental disorders in Taiwan. Psychological Medicine 16(2): 415-422.

Cohen, S., B.H. Gottlieb, and L.G. Underwood. 2000. Social relationships and health. In Social support measurement and intervention: A guide for health and social scientists, ed. S. Cohen, L.G. Underwood, and B.H. Gottlieb, 3-25. New York: Oxford University Press.

Cohen, S., R. Mermelstein, T. Karmarck, and H.M. Hoberman. 1985. Measuring the functional components of social support. In Social support: Theory, research and application, ed. I.G. Sarason, and B.R. Sarason, 73-94. The Hague, The Netherlands: Martinus Nijhoff.

Cone, J.E., J. Li, E. Kornblith, V. Gocheva, S.D. Stellman, A. Shaikh, R. Schwarzer, and R.M. Bowler. 2015. Chronic probable PTSD in police responders in the World Trade Center health registry ten to eleven years after 9/11. American Journal of Industrial Medicine 58(5): 483-493.

Connor, K.M., and J.R.T. Davidson. 2003. Development of a new resilience scale: The Connor-Davidson resilience scale $(\mathrm{CD}$ RISC). Depression and Anxiety 18(2): 76-82.

Curtona, C.E., and D.W. Russell. 1990. Type of social support and specific stress: Toward a theory of optimal matching. In Social support: An interactional view, ed. I.G. Sarason, and G.R. Pierce, 319-366. Oxford: John Wiley.

de Terte, I., C. Stephens, and L. Huddleston. 2014. The development of a three part model of psychological resilience. Stress and Health 30(5): 416-424.

Derogatis, L.R., and P.A. Cleary. 1977. Factorial invariance across genderfor the primary symptom dimensions of the SCL-90. British Journal of Clinical Psychology 16(4): 347-356.

Derogatis, L.R., and P.M. Spencer. 1993. Brief symptom inventory (BSI). Upper Saddle River, NJ: Pearson.

Diener, E.D., R.A. Emmons, R.J. Larsen, and S. Griffin. 1985. The satisfaction with life scale. Journal of Personality Assessment 49(1): 71-75.

Dougall, A.L., K.B. Hyman, M.C. Hayward, S. McFeeley, and A. Baum. 2001. Optimism and traumatic stress: The importance of social support and coping. Journal of Applied Social Psychology 31(2): 223-245.

Ehring, T., S. Razik, and P.M.G. Emmelkamp. 2011. Prevalence and predictors of posttraumatic stress disorder, anxiety, depression, and burnout in Pakistani earthquake recovery workers. Psychiatry Research 185(1-2): 161-166.

Feder, A., N. Mota, R. Salim, J. Rodriguez, R. Singh, J. Schaffer, C.B. Schechter, L.M. Cancelmo, et al. 2016. Risk, coping and PTSD symptom trajectories in World Trade Center responders. Journal of Psychiatric Research 82: 68-79. 
Field, A.P., and R. Gillett. 2010. How to do a meta-analysis. British Journal of Mathematical and Statistical Psychology 63(3): 665-694.

Fleming, R., A. Baum, M.M. Gisriel, and R.J. Gatchel. 1982. Mediating influences of social support on stress at Three Mile Island. Journal of Human Stress 8(3): 14-23.

Foa, E.B., D.S. Riggs, C.V. Dancu, and B.O. Rothbaum. 1993. Reliability and validity of a brief instrument for assessing posttraumatic stress disorder. Journal of Traumatic Stress 56(4): 459-473.

Folkman, S., and R.S. Lazarus. 1988. Ways of coping questionnaire. Washington, DC: Consulting Psychologists Press.

Fullerton, C.S., J.B.A. McKibben, D.B. Reissman, T. Scharf, K.M. Kowalski-Trakofler, J.M. Shultz, and R.J. Ursano. 2013. Posttraumatic stress disorder, depression, and alcohol and tobacco use in public health workers after the 2004 Florida hurricanes. Disaster Medicine and Public Health Preparedness 7(1): 89-95.

Fullerton, S., R.J. Ursano, X. Liu, J.B.A. McKibben, L. Wang, and D.B Reissman. 2015. Depressive symptom severity and community collective efficacy following the 2004 Florida hurricanes. PloS One 10(6): e0130863.

Gardner, R.C. 2010. Research design-Topic 10: Multiple regression and multiple correlation. http://publish.uwo.ca/ gardner/DataA nalysisDotCalm/Lectures2010/TOPIC10c.pdf. Accessed 24 May 2018.

Goldberg, P. 1972. The detection of psychiatric illness by questionnaire: A technique for the identification and assessment of nonpsychotic illness. Oxford: Oxford University Press.

Goldmann, E., and S. Galea. 2014. Mental health consequences of disasters. Annual Review of Public Health 35: 169-183.

Guha-Sapir, D., P. Hoyois, P. Wallemacq, and R. Below. 2016. Annual disaster statistical review 2016: The numbers and trends. Brussels: CRED.

Halbesleben, J.R.B. 2006. Sources of social support and burnout: A meta-analytic test of the conservation of resources model. Journal of Applied Psychology 91(5): 1134-1145.

Hamblen, J.L., F.H. Norris, K.A. Symon, and T.E. Bow. 2017. Cognitive behavioral therapy for postdisaster distress: A promising transdiagnostic approach to treating disaster survivors. Psychological Trauma: Theory, Research, Practice and Policy 9(S1): 130-136.

Harada, N., J. Shigemura, M. Tanichi, K. Kawaida, S. Takahashi, and F. Yasukata. 2015. Mental health and psychological impacts from the 2011 Great East Japan Earthquake disaster: A systematic literature review. Disaster and Military Medicine 1(1): Article 17.

Hedges, L.V., and I. Olkin. 1985. Statistical methods for metaanalysis. Orlando, FL: Academic Press.

Hedges, L.V., and J.L. Vevea. 1998. Fixed- and random-effects models in meta-analysis. Psychological Methods 3(4): 486-504.

Hobfoll, S.E., and J.P. Stokes. 1988. The process and mechanics of social support. In Handbook of personal relationships: Theory, research and interventions, ed. S. Duck, D.F. Hay, S.E. Hobfoll, W. Ickes, and B.M. Montgomery, 497-517. Oxford: John Wiley.

Hobfoll, S.E., P. Watson, C.C. Bell, R.A. Bryant, M.J. Brymer, M.J. Friedman, M. Friedman, P.R. Berthold, et al. 2007. Five essential elements of immediate and mid-term mass trauma intervention: Empirical evidence. Psychiatry 70(4): 283-315.

Holahan, C.J, and R.H. Moos. 1981. Social support and psychological distress: A longitudinal analysis. Journal of Abnormal Psychology 90(4): 365-370.

Horowitz, M., N. Wilner, and W. Alvarez. 1979. Impact of event scale: A measure of subjective stress. Psychosomatic Medicine 41(3): 209-218

Huang, J., Q. Liu, J. Li, X. Li, J. You, L. Zhang, C. Tian, and R. Luan. 2013. Post-traumatic stress disorder status in a rescue group after the Wenchuan Earthquake relief. Neural Regeneration Research 8(20): 1898-1906.

Jenkins, S.R. 1996. Social support and debriefing efficacy among emergency medical workers after a mass shooting incident. Journal of Social Behavior \& Personality 11(3): 477-492.

Jenkins, S.R. 1997. Coping and social support among emergency dispatchers: Hurricane Andrew. Journal of Social Behavior \& Personality 12(1): 201-216.

Jose, P.E. 2013. Doing statistical mediation \& moderation. New York: Guilford Press.

Joseph, S., B. Andrews, R. Williams, and W. Yule. 1992. Crisis support and psychiatric symptomatology in adult survivors of the Jupiter cruise ship disaster. British Journal of Clinical Psychology 31(1): 63-73.

Kaniasty, K., and F.H. Norris. 1993. A test of the social support deterioration model in the context of natural disaster. Journal of Personality and Social Psychology 64(3): 395-408.

Kaniasty, K., and F.H. Norris. 1995. Mobilization and deterioration of social support following natural disasters. Current Directions in Psychological Science 4(3): 94-98.

Kaniasty, K., and F.H. Norris. 2008. Longitudinal linkages between perceived social support and posttraumatic stress symptoms: Sequential roles of social causation and social selection. Journal of Traumatic Stress 21(3): 274-281.

Kaniasty, K., and F.H. Norris. 2009. Distinctions that matter: Received social support, perceived social support, and social embeddedness after disasters. In Mental health and disasters, ed. Y. Neria, S. Galea, and F.H. Norris, 175-200. New York: Cambridge University Press.

Keane, T.M., J.M. Caddell, and K.L. Taylor. 1988. Mississippi scale for combat-related posttraumatic stress disorder: Three studies in reliability and validity. Journal of Consulting and Clinical Psychology 56(1): 85-90.

Kulka, R.A., W.E. Schlenger, J.A. Fairbank, R.L. Hough, B.K. Jordan, C.R. Marmar, and D.S. Weiss. 1988. Contractual report of findings from the national Vietnam veterans' readjustment study: Volumes 1-4. Research Triangle Park, NC: Research Triangle Institute.

Leppma, M., A. Mnatsakanova, K. Sarkisian, O. Scott, L. Adjeroh, M.E. Andrew, J.M. Violanti, and E.C. McCanlies. 2017. Stressful life events and posttraumatic growth among police officers: A cross-sectional study. Stress and Health 34(1): $175-186$.

Marmar, C.R., S.E. McCaslin, T.J. Metzler, S. Best, D.S. Weiss, J. Fagan, A. Liberman, N. Pole, C. Otte, R. Yehuda, D. Mohr, and T. Neylan. 2006. Predictors of posttraumatic stress in police and other first responders. Annals of the New York Academy of Sciences 1071: 1-18.

Maslach, C., S.E. Jackson, and M.P. Leiter. 1986. Maslach burnout inventory. Palo Alto, CA: Mind Garden, Inc.

McCullough, M.E., R.A. Emmons, and J.A. Tsang. 2002. The grateful disposition: A conceptual and empirical topography. Journal of Personality and Social Psychology 82(1): 112-117.

Moher, D., A. Liberati, J. Tetzlaff, D.G. Altman, and The PRISMA Group. 2009. Preferred reporting items for systmatic reviews and meta-analyses: The PRISMA statement. PLoS Med 6(7): e1000097.

Mollica, R.F., Y. Caspi-Yavin, P. Bollini, T. Truong, S. Tor, and J. Lavelle. 1992. The Harvard trauma questionnaire: Validating a cross-cultural instrument for measuring torture, trauma, and posttraumatic stress disorder in Indochinese refugees. Journal of Nervous \& Mental Disease 180(2): 111-116.

Mumford, D.B., M. Ayub, R. Karim, N. Izhar, A. Asif, and J.T. Bavington. 2005. Development and validation of a questionnaire for anxiety and depression in Pakistan. Journal of Affective Disorders 2: 175-182. 
Mumford, D.B., J.T. Bavington, K.S. Bhatnagar, Y. Hussain, S. Mirza, and S.S. Naraghi. 1991. The Bradford somatic inventory: A multi-ethnic inventory of somatic symptoms reported by anxious and depressed patients in Britan and the Indo-Pakistan subcontinent. The British Journal of Psychiatry 158(3): 379-386.

Murphy, S.A., L.C. Johnson, and R.D. Beaton. 2004. Fire fighters' cognitive appraisals of job concerns, threats to well-being, and social support before and after the terrorist attacks on September 11, 2001. Journal of Loss and Trauma 9(3): 269-283.

Norris, F.H., and C.L. Elrod. 2006. Psychosocial consequences of disaster: A review of past research. In Methods for disaster mental health research, ed. F.H. Norris, S. Galea, M.J. Friedman, and P.J. Watson, 20-42. New York: Guilford Press.

Norris, F.H., M.J. Friedman, P.J. Watson, C.M. Byrne, E. Diaz, and K. Kaniasty. 2002. 60,000 disaster victims speak: Part I. An empirical review of the empirical literature, 1981-2001. Psychiatry 65(3): 207-239.

Ozer, E.J., S.R. Best, T.L. Lipsey, and D.S. Weiss. 2003. Predictors of posttraumatic stress disorder and symptoms in adults: A metaanalysis. Psychological Bulletin 129(1): 52-73.

Perilla, J.L., F.H. Norris, and E.A. Lavizzo. 2002. Ethnicity, culture, and disaster response: Identifying and explaining ethnic differences in PTSD six months after Hurricane Andrew. Journal of Social and Clinical Psychology 21(1): 20-45.

Pietrzak, R.H., A. Feder, R. Singh, C.B. Schechter, E.J. Bromet, C.L. Katz, D.B. Reissman, F. Ozbay, et al. 2014. Trajectories of PTSD risk and resilience in World Trade Center responders: An 8-year prospective cohort study. Psychological Medicine 44(1): 205-219.

Prati, G., and L. Pietrantoni. 2010. The relation of perceived and received social support to mental health among first responders: A meta-analytic review. Journal of Community Psychology 38(3): 403-417.

Radloff, L.S. 1977. The CES-D scale: A self-report depression scale for research in the general population. Applied Psychological Measurement 1(3): 385-401.

Rini, C., and C.D. Dunkel Schetter. 2010. The effectiveness of social support attempts in intimate relationships. In Support processes in intimate relationships, ed. K. Sullivan and J. Davila, 26-67. New York: Oxford University Press.

Ritvo, P.G., J.S. Fischer, D.M. Miller, H. Andrews, D.W. Paty, and N.G. LaRocca. 1997. Multiple sclerosis quality of life inventory: A user's manual. New York: National Multiple Sclerosis Society.

Schaufeli, W.B., A.B. Bakker, and M. Salanova. 2006. The measurement of work engagement with a short questionnaire: A cross-national study. Educational and Psychological Measurement 66(4): 701-716.

Schenk, E.J., J. Yuan, L.D. Martel, G.Q. Shi, K. Han, and X. Gao. 2016. Risk factors for long-term post-traumatic stress disorder among medical rescue workers appointed to the 2008 Wenchuan Earthquake response in China. Disasters 41(4): 788-802.

Schwarzer, R., R.M. Bowler, and J.E. Cone. 2014. Social integration buffers stress in New York police after the 9/11 terrorist attack. Anxiety, Stress, and Coping 27(1): 18-26.

Schwarzer, R., J.E. Cone, J. Li, and R.M. Bowler. 2016. A PTSD symptoms trajectory mediates between exposure levels and emotional support in police responders to 9/11: A growth curve analysis. BMC Psychiatry 16: Article 201.
Shepherd, D., D. McBride, and K. Lovelock. 2017. First responder well-being following the 2011 Canterbury Earthquake. Disaster Prevention and Management 26(3): 286-297.

Sherbourne, C.D., and A.L. Stewart. 1991. The MOS social support survey. Social Science and Medicine 32(6): 705-714.

Stamm, B.H. 2005. The professional quality of life scale. Pocatello: Idaho State University.

Stamm, B.H., M.A. Rudolph, A. Smith, and E.M. Varra. 1998. Life status review: Monitoring biopsychosocial risk factors in traumatic stress. http://www.isu.edu/ bhstamm/pdf/LSR_pos.pdf. Accessed 24 May 2018.

Stephens, C., N. Long, and I. Miller. 1997. The impact of trauma and social support on posttraumatic stress disorder: A study of New Zealand police officers. Journal of Criminal Justice 25(4): 303-314.

Tak, S.W., R. Driscoll, B. Bernard, and C. West. 2007. Depressive symptoms among firefighters and related factors after the response to Hurricane Katrina. Journal of Urban Health 84(2): $153-161$.

Tam, C.W.C., E.P.F. Pang, L.C.W. Lam, and H.F.K. Chiu. 2004. Severe acute respiratory syndrome (SARS) in Hong Kong in 2003: Stress and psychological impact among frontline healthcare workers. Psychological Medicine 34(7): 1197-1204.

Tedeschi, R.G., and L.G. Calhoun. 1996. The posttraumatic growth inventory: Measuring the positive legacy of trauma. Journal of Traumatic Stress 9(3): 455-471.

Timmerman, I.G.H., E.S. Emanuels-Zuurveen, and P.M.G. Emmelkamp. 2000. The social support inventory (SSI): A brief scale to assess perceived adequacy of social support. Clinical Psychology and Psychotherapy 7(5): 401-410.

Vreven, D.L., D.M. Gudanowski, L.A. King, and D.W. King. 1995. The civilian version of the Mississippi PTSD scale: A psychometric evaluation. Journal of Traumatic Stress 8(1): 91-109.

Wagnild, G., and H.M. Young. 1993. Development and psychometric evaluation of the resilience scale. Journal of Nursing Measurement 1(2): 165-178.

Wall, T.D., P.R. Jackson, and S. Mullarkey. 1995. Further evidence on some new measures of job control, cognitive demand and production responsibility. Journal of Organizational Behavior 16(5): 431-455.

Warr, P., J. Cook, and T. Wall. 1979. Scales for the measurement of some work attitudes and aspects of psychological well-being. Journal of Occupational and Organizational Psychology 52(2): $129-148$.

Weathers, F.W., B.T. Litz, D.S. Herman, J.A. Huska, and T.M. Keane. 1993. The PTSD checklist (PCL): Reliability, validity, and diagnostic utility. In Proceedings of the Annual Conference of the International Society for Traumatic Stress Studies, 25 Oct 1993, San Antonio, Texas.

Weiss, D.S. 2007. The impact of event scale: Revised. In Crosscultural assessment of psychological trauma and PTSD, ed. J.P. Wilson, and C.S. Tang, 219-238. New York: Springer.

Weiss, D.S., C.R. Marmar, T.J. Metzler, and H.M. Ronfeldt. 1995. Predicting symptomatic distress in emergency services personnel. Journal of Consulting and Clinical Psychology 63(3): 361-368.

Wu, K.K., and K.S. Chan. 2003. The development of the Chinese version of impact of event scale-revised (CIES-R). Social Psychiatry and Psychiatric Epidemiology 38(2): 94-98. 